\title{
Investigating on the Nuclear Obscuration in Two Types of Seyfert 2 Galaxies
}

\author{
X. W. Shu, J. X. Wang, P. Jiang, L. L. Fan, and T. G. Wang \\ Center for Astrophysics, University of Science and Technology of China, Hefei, Anhui \\ 230026, P. R. China \\ Joint Institute of Galaxies and Cosmology, USTC and SHAO, CAS \\ jxw@ustc.edu.cn
}

\begin{abstract}
We build a large sample of Seyfert 2 galaxies (Sy2s) with both optical spectropolarimetric and X-ray data available, in which 29 Sy2s with the detection of polarized broad emission line (PBL) and 25 without. We find that for luminous Sy2s with $\mathrm{L}_{[\mathrm{O} \text { III }]}>10^{41} \mathrm{erg} \mathrm{s}^{-1}$, sources with PBL have smaller X-ray absorption column density comparing with those without PBL (at $92.3 \%$ confidence level): most of the Sy2s with $\mathrm{N}_{\mathrm{H}}<10^{23.8} \mathrm{~cm}^{-2}$ show PBL (86\%, 12 out 14), while the fraction is much smaller for sources with heavier obscuration (54\%, 15 out 28). The confidence level of the difference in absorption bounces up to $99.1 \%$ while using the "T" ratio $\left(\mathrm{F}_{2-10 \mathrm{keV}} / \mathrm{F}_{[\mathrm{O} \text { III] }}\right)$ as an indicator. We rule out observation or selection bias as the origin for the difference. Our results, for the first time with high statistical confidence, show that, in additional to the nuclei activity, the nuclear obscuration also plays an important role in the visibility of PBL in Sy2s. These results can be interpreted in the framework of the unified model. We can reach these results in the unified model if: a) the absorption column density is higher at large inclinations and b) the scattering region is obscured at large inclinations.
\end{abstract}

Subject headings: galaxies: active — galaxies: Seyfert — polarization

\section{Introduction}

The AGN unification model proposes that Seyfert 1 and 2 galaxies (Sy1s and Sy2s hereafter) are intrinsically the same objects, and the absence of broad emission lines in Sy2s is ascribed to the obscuration along the line of sight by a pc-scale dusty torus (see 
the review by Antonucci 1993). The most convincing evidence is the detection of polarized broad emission lines (hereafter PBL) in some Seyfert 2 galaxies (Antonucci \& Miller 1985; Tran 1995; Heisler, Lumsden \& Bailey 1997; Moran et al. 2000; Lumsden et al. 2001; Tran 2001). Similarly infrared (IR) observations showed the existence of obscured broad line regions (BLRs) in Sy2s (Veilleux, Goodrich \& Hill 1997). Further evidence supporting the unification model comes from X-ray observations of Sy2s which show large amounts of obscuration, typically above $10^{23} \mathrm{~cm}^{-2}$ (e.g., Turner et al. 1997; Bassani et al. 1999).

Despite observations do generally support orientation based unification model, only $50 \%$ of Sy2s show broad lines in the polarized spectrum (e.g. Tran 2001, Gu \& Huang 2002). With an optical spectropolarimetric study of a well-defined and statistically complete IRAS 60- $\mu$ m selected Sy2 sample, Heisler et al. (1997) found a relationship between the detectability of polarized broad $\mathrm{H} \alpha$ and the IRAS $f_{60} / f_{25}$ flux ratio that only those galaxies with warm IRAS colors $\left(f_{60} / f_{25}<4.0\right)$ show PBL. Heisler et al. suggested that the detectability of PBL simply depends on the inclination of the torus to the line of sight: in a Sy2 with the torus highly inclined, cooler infrared color is expected, and the broad-line scattering screen could also be obscured.

A simple prediction of the inclination-related model is that Sy2s without PBL (hereafter NPBL Sy2s) should show higher absorption column density since they are more inclined than the Sy2s with PBL (hereafter PBL Sy2s). However, following studies have claimed no difference in the absorption column density between two types of Sy2s (Alexander 2001; Tran 2001, 2003). Furthermore, as Alexander (2001, also see Lumsden et al 2001; Tran 2001, 2003; Gu \& Huang 2002) pointed out, the difference in the $I R A S f_{60} / f_{25}$ flux ratio is not an good indicator of the inclination but the relative strength of galactic and Seyfert emission. These studies (also see Cheng et al. 2002; Lumsden \& Alexander 2001), instead, have shown that the presence of PBL in Sy2s depends on the AGN luminosity: Sy2s with PBL have higher luminosity comparing with Sy2s without PBL. Explanations to the observational results include: a) The contribution from the host galaxy or from a circumnuclear starburst would dilute the nuclear optical spectrum, making the detection of PBL more difficult for Sy2s with lower luminosity (Alexander 2001; Gu et al. 2001); b) Alternatively, Tran (2001, 2003, also see Yu \& Hwang 2005) suggested that at least some of the Sy2s without PBL are powered by starburst rather than accretion onto a supermassive black hole, therefore, the BLRs simply do not exist; c) More luminous sources tend to have large scaleheight of the scattering region thus increasing the visibility of PBL (Lumsden \& Alexander 2001); d) Nicastro, Martocchia \& Matt (2003) have argued that at very low accretion rates (and therefore lower luminosities) the clouds of the BLRs would cease to exist and the absence of PBL in Sy2s is consistent to their low accretion rates. e) In the case of low luminosity nuclei, the adjacent bright sources can easily outshine the nuclear flux and the $\mathrm{N}_{\mathrm{H}}$ derived 
from X-ray spectrum may be underestimated (e.g. Georgantopoulos \& Zezas 2003). f) The large-scale dusty environment (Panessa \& Bassani 2002) or complex and variable obscuring material (Matt 2000a; Risaliti 2002) may to some extent affect the appearance of PBL in Sy2s. g) Long term large amplitude variation in the nuclei activity could vary the PBL flux and thus the detectability of PBL (Lumsden et al. 2004).

Meanwhile, it's worth to note that there are also weak evidences showing different absorption in two types of Sy2s: Gu, Maiolino \& Dultzin-Hacyan (2001) found slightly (but not statistically conclusive) lower $\mathrm{N}_{\mathrm{H}}$ for PBL Sy2s, and Lumsden, Alexander \& Hough (2004) found a considerably higher detection rate of scattered broad $\mathrm{H}_{\alpha}$ in a small sample of Compton-thin Sy2s. Note one must be cautious while comparing the fraction of PBL Sy2s between samples since the luminosity might have played a major role. These evidences suggest that besides the AGN luminosity, the X-ray absorption column density also plays a role in the visibility of PBL in Sy2s. We point out that the role of the absorption (if exists) could reveal itself in a luminous Sy2 sample where the influence of luminosity on the visibility of PBL is weak enough.

In this paper we revisit the issue of whether the nuclear obscuration in Seyfert 2 galaxies affects the visibility of PBL by focusing on luminous Sy2s. The launch of the Chandra X-ray Observatory in 1999 and XMM-Newton in 2000 open a new era of X-ray astronomy. New Chandra and XMM-Newton observations have significantly enlarged the sample of Sy2s with both spectropolarimetric and X-ray observations available, and also provide more reliable X-ray measurements thanks to their much higher spatial resolution and better sensitivity. In this paper, we present a large sample of Sy2s, for which both the spectropolarimetric and X-ray data are available, to probe the nuclear obscuration for PBL/NPBL Sy2s. Our sample consists of 29 PBL Sy2s and 25 NPBL Sy2s. Among them 8 Chandra and 30 XMMNewton observations are available either from literature or from archive. Throughout this paper we use the cosmological parameters $\mathrm{H}_{0}=70 \mathrm{~km} \mathrm{~s}^{-1} \mathrm{Mpc}^{-1}, \Omega_{m}=0.27$ and $\Omega_{\lambda}=0.73$.

\section{Sample selection}

We collect all Sy2s with spectropolarimetric observations from literature (from 1985 to 2006, see table 1). The spectropolarimetric data are mainly from several large surveys including: the infrared-selected sample of Heisler et al. (1997), the far infrared flux and luminosity limited sample of Lumsden et al. (2001), the distance limited sample of Moran et al. (2000, 2001), the heterogeneous optical and mid infrared selected sample of Tran et al. (2001), and infrared color selected sample of Young et al. (1996). We then exclude NGC 
2992, NGC 5506, NGC 5252, NGC 7314, MCG -3-34-64 and Mrk 334 from discussion due to their intermediate classification (i.e. Sy1.8s, 1.9s) in NED. To avoid luminosity selection bias due to the redshift difference when comparing properties between the PBL and NPBL Sy2s, we confine our sample within $\mathrm{z}<0.06$. We collect $[\mathrm{O}$ III $] \lambda 5007$ and X-ray data from literature, and present spectra analysis of archive Chandra/XMM-Newton data for 8 sources in $\S 3$. The result from most recent observation is adopted when two or more observations exist. Note in the table, there are 8 upper limits in the hard X-ray flux due to X-ray non-detection in the hard band and their X-ray absorption column densities in literature were estimated either from soft band X-ray data or the strength of their X-ray emission relative to optical band. We exclude these $\mathrm{N}_{\mathrm{H}}$ from our following analysis. NGC 4117 is also excluded since its [O III] $\lambda 5007$ flux is not available from literature. This leaves a sample composing of 292 Sy2s with PBL, and 25 Sy2s without PBL, for which both the spectropolarimetric and X-ray data are available. Fig. 1 shows the redshift against the luminosity of extinction-corrected [O III] $\lambda 5007$ emission for PBL and NPBL Sy2s in the sample. As previous studies have shown, we clearly see higher luminosities for PBL Sy2s (with a confidence level of 99.7\%), indicating the nulear activity plays a major role in the visibility of PBL in Sy2s.

The optical and X-ray data are presented in Table 1. The table lists, in turn, the name of the galaxy, redshift $z$ as reported in NED, the spectropolarimetric properties, the corresponding references, the extinction-corrected flux of [O III $] \lambda 5007$ emission in units of $10^{-12} \mathrm{erg} \mathrm{s}^{-1} \mathrm{~cm}^{-2}$, the luminosity of extinction-corrected [O III] $\lambda 5007$ emission in units of $\operatorname{erg~s}^{-1}$, the references for the [O III] $\lambda 5007$ emission, the observed rest frame hard X-ray (2-10 keV) flux in units of erg $\mathrm{s}^{-1}$, the X-ray absorption column density $\left(\mathrm{N}_{\mathrm{H}}\right)$ in units of $\mathrm{cm}^{-2}$, the equivalent width (EW) of the fluorescence iron line in units of $\mathrm{eV}$, the references for the X-ray properties. The luminosity of extinction-corrected [O III $\lambda \lambda 5007$ emission is given as $\left.\mathrm{L}_{[\mathrm{O}} \mathrm{III}\right]=4 \pi \mathrm{D}^{2} \mathrm{~F}_{[\mathrm{O}}^{\text {cor }}$ III $]$, where $\mathrm{F}_{[\mathrm{O} \text { III }]}^{\text {cor }}$ is the extinction-corrected flux of [O III $] \lambda 5007$ emission derived from the relation (Bassani et al. 1999)

$$
F_{[\mathrm{O} \text { III }]}^{\mathrm{cor}}=F_{[\mathrm{O} \text { III }]}^{\mathrm{obs}}\left(\frac{\left(\mathrm{H}_{\alpha} / \mathrm{H}_{\beta}\right)_{\mathrm{obs}}}{\left(\mathrm{H}_{\alpha} / \mathrm{H}_{\beta}\right)_{0}}\right)^{2.94}
$$

We assume an intrinsic Balmer decrement $\left(\mathrm{H}_{\alpha} / \mathrm{H}_{\beta}\right)_{0}=3.0$.

\footnotetext{
${ }^{1}$ Among the six sources, NGC 5506 and Mrk 334 do not show PBL in the polarized spectra and the rest four do. Including these sources in our sample does not make significant difference to our major results.

${ }^{2}$ For one galaxy IRAS $04385-0828$, the value of $\mathrm{N}_{\mathrm{H}}$ is unavailable, but we can get the reference to hard X-ray flux from Polletta et al. (1996). When using the "T" ratio for analysis, the number for PBL Sy2s is 30.
} 


\section{X-ray spectral analysis}

In this section, we report the results of X-ray spectral fitting to archive Chandra and XMM-Newton spectra of 8 Sy2s in the sample. The data were reduced using CIAO 3.2.2 and XMMSAS 6.5.0 respectively. The size of each source on the detector was estimated in order to determine appropriate source extraction regions, typically $\sim 2$ " radius (Chandra) or $\sim 30 "$ (XMM-Newton) for on-axis point sources. The background spectra were extracted from source-free annulus around the source. The spectra of each galaxy were binned to a minimum of 1 counts per bin and we adopt the C-statistic (Cash 1979) for minimization. Spectral fits were performed using XSPEC version 11.2 in the $0.5-8 \mathrm{keV}$ band. All the quoted errors are $90 \%$ confidence range for one parameter of interest.

Each spectrum was initially fitted with a simple model consisting of a powerlaw plus Galactic and intrinsic neutral absorption. In many cases this simple parameterization is not sufficient to model the whole $0.5-8 \mathrm{keV}$ spectrum. Residuals often show a soft excess on top of the powerlaw. The soft excess is fitted here as a scattered powerlaw component (with the same powerlaw slope but no intrinsic absorption). The possible presence of a narrow emission line centered at $6.4 \mathrm{keV}$ originating from neutral iron has also been checked, and modeled with a single Gaussian line.

We note that in Compton-thick sources with $\mathrm{N}_{H}>10^{24} \mathrm{~cm}^{-2}$, the transmitted component is heavily suppressed below $10 \mathrm{keV}$ and the spectrum observed in the $2-10 \mathrm{keV}$ band might be dominated by the reflection component (Matt et al. 2000b). In this paper, NGC 34, NGC 3982, Mrk 573, Mrk 1066 are classified as Compton-thick based on their large Fe $\mathrm{K} \alpha \mathrm{EW}$ (> $>1 \mathrm{keV}$ except for NGC 34, see notes in Appendix) and small $\mathrm{F}_{2-10 \mathrm{keV}} / \mathrm{F}_{\text {[O III] }}$ ratios (<0.1) (Maiolino et al. 1998, Bassani et al. 1999, Guainazzi et al. 2005b). For Compton-thick sources, we use the reflection model (pexrav model in XSPEC; Magdziarz \& Zdziarski 1995) for spectrum fitting.

Given the purpose of this work (to obtain a proper description of the spectra in terms of absorption, 2-10 keV flux, and Fe K line intensity), these simple parameterizations yield adequate fits to all the spectra presented here. The best-fit spectral parameters are listed in Table 2 and notes on individual objects are given in Appendix.

\section{4. different obscuration in two types of Sy2s}

In Fig. 2 (b), we plot the luminosity of extinction-corrected [O III] $\lambda 5007$ emission versus $\mathrm{N}_{\mathrm{H}}$. The separation is apparent for two types of Sy2s. The diagram can be roughly divided into three regions with the boundaries at $\mathrm{N}_{\mathrm{H}}=10^{23.8} \mathrm{~cm}^{-2}$ and $\mathrm{L}_{[\mathrm{O} \mathrm{III}]}=10^{41} \mathrm{erg} \mathrm{s}^{-1}$. For 
the luminous Sy2s (with $\mathrm{L}_{[\mathrm{O} \text { III }]}>10^{41} \mathrm{erg} \mathrm{s}^{-1}$ ), we can clearly see that most of the Sy2s with $\mathrm{N}_{\mathrm{H}}<10^{23.8} \mathrm{~cm}^{-2}$ show PBL $(86 \%, 12$ out 14$)$, while the fraction is much smaller for sources with heavier obscuration $(54 \%, 15$ out 28$)$. For the Sy2s with lower [O III] luminosity $(<$ $\left.10^{41} \mathrm{erg} \mathrm{s}^{-1}\right)$, only a small fraction show PBL $(17 \%, 2$ out of 12$)$, and due to the limited number of sources, we are not able to tell if the fraction of PBL sources depends on the X-ray absorption at lower luminosity.

In Fig. 3 (left panel) we plot the $\mathrm{N}_{\mathrm{H}}$ distributions for all Sy2s with/out PBL. Since there are 11 censored data (lower limits) among PBL Sy2s and 10 among NPBL Sy2s, we use the survival analysis methods ASURV (Feigelson \& Nelson 1985) for statistical analysis. We find little difference (with a confidence level of $66.5 \%$ of the difference, see table 3 ) in $\mathrm{N}_{\mathrm{H}}$ between PBL/NPBL Sy2s and the mean values of $\log \mathrm{N}_{\mathrm{H}}$ (in units of $\mathrm{cm}^{-2}$ ) are $23.755 \pm 0.19$ and 23.852 \pm 0.274 , respectively (for NGC 4501 and NGC 7590 we adopt the $\mathrm{N}_{\mathrm{H}}$ upper limits as the measured values since ASURV could not deal with the case which contains both upper and lower limits). However, if we only consider the luminous Sy2s with $\mathrm{L}_{[\mathrm{O}}$ III] $>10^{41} \mathrm{erg} \mathrm{s}^{-1}$, K-S test shows that the probability for two samples to be extracted from the same parent population is about 7.7\%, and the mean values of $\log \mathrm{N}_{\mathrm{H}}$ are $23.739 \pm 0.212$ and $24.428 \pm 0.192$, respectively (Fig. 3, right panel). The results suggested that for luminous Sy2s in our sample, sources without PBL show larger obscuration than those with PBL with a confidence level of $92.3 \%$.

To further examine if obscuration plays a role in the detection/visibility of PBL in Sy2s, we explore other potential measures of obscuration. By studying a large sample of Sy2s, Bassani et al. (1999) found that the "T" ratio $\mathrm{F}_{2-10 \mathrm{keV}} / \mathrm{F}_{[\mathrm{O} \text { III] }}$ is a good indicator of nuclear obscuration. In particular, it is anticorrelated with both the column density $\mathrm{N}_{\mathrm{H}}$ and the Fe $\mathrm{K} \alpha$ line EW, and that these quantities can be used as probes of the obscuration to the center of the AGN. In Fig. 2 (c, d) we plot the Fe K $\alpha$ line EW and "T" ratio versus the luminosity of extinction-corrected [O III] $\lambda 5007$ emission. Similar patterns as seen in Fig. 2 (b) are also obvious that for luminous Sy2s, the NPBL sources tend to be more obscured. Fig. 4 shows the "T" ratio distributions for all Sy2s (left) and for luminous objects only (right). A K-S test shows that the possibility for these two samples to be extracted from the same parent population is about $25 \%$. The mean value of the log $\mathrm{T}$ are $-0.087 \pm 0.145$ and $-0.342 \pm 0.217$ for Sy2s with and without PBL respectively. Similarly the confidence level for the difference is much higher (at level of 99.1\%) for luminous Sy2s only. Turning to the Fe K $\alpha$ line EW, K-S tests also confirms that for luminous Sy2s the difference between the two sample is present (at $95.3 \%$ level) with available data. The mean values of $\log$ $\mathrm{EW}(\mathrm{Fe})$ are 2.626 \pm 0.107 and 2.999 \pm 0.066 respectively. After examining three independent indicators for obscuration, we conclude that for luminous Sy2s, sources without PBL have higher obscuration than those with PBL, confirming the suggestion that the obscuration does 
play an important role in the detectability/visibility of PBL. The results from K-S tests and average values for $\mathrm{N}_{\mathrm{H}}$, "T" ratio and FeK line EW are summarized in table 3.

\section{Discussion}

It's clear that, as many previous studies have shown, PBL Sy2s have higher luminosities than NPBL Sy2s (see Fig. 1 \& 2), indicating the primary determinant of PBL visibility is the nuclei luminosity. In this paper, by focusing on luminous Sy2s, we find that the nuclei obscuration also plays an important role in the visibility of PBL. For Sy2s with $\mathrm{L}_{[\mathrm{O} \text { III] }}>$ $10^{41} \mathrm{erg} \mathrm{s}^{-1}$ in our sample, we find that NPBL Sy2s have higher X-ray column density than PBL Sy2s at a significant level of 92.3\%. While using the "T" ratio or the Fe K line EW as indicator of nuclear obscuration, the confidence level of the difference in obscuration gets even higher (99.1\% and 95.3\% respectively). Our results are consistent with Lumsden et al. (2004) who reported higher detection rate of PBL in Compton-thin Sy2s, but with much higher confidence level. Consistent with previous studies, most (83\%) of the less luminous Sy2s $\left.\left(\mathrm{L}_{[\mathrm{O}} \mathrm{III}\right]<10^{41} \mathrm{erg} \mathrm{s}^{-1}\right)$ do not show PBL, the nature of which is still unclear (see $\S 1$ ) and is beyond the scope of this paper. We also demonstrate that since most of the less luminous Sy2s do not show PBL independent of absorption, adding them to the sample weakens the difference in obscuration found in the luminous sample. This explains why previous studies, which did not exclude less luminous sources, found no difference in absorption.

\subsection{Selection effect?}

It is worth stressing whether the difference in the nuclear obscuration between two types of Sy2s could be due to possible observational and sample selection bias. We note our sample is an amalgamation of different observations with diverse quality of spectropolarimetric data. We first examine whether the non-detections of PBL in the sample are due to the weakness/lack of PBL or due to the limited sensitivities of the spectropolarimetric data. Lumsden et al. (2001) showed that the $\mathrm{S} / \mathrm{N}$ in their sample is sufficient for all but 2 of the NPBL S2s and attributed the nondetections to significantly weaker scattered flux. Tran (2003) pointed out that the distributions of [O III] flux, which is a good indicator of the strength of the Seyfert nucleus, are virtually the same between two types of Sy2s in his sample, suggesting that the nondetections are not likely due to the detection limit of the survey. The sensitivity of Moran's sample (2001) is found to be even better than that of the other samples mentioned above ( $\mathrm{Gu} \&$ Huang 2002). In Fig. 5, we plot the "T" ratio vs. the extinction corrected [O III] flux for our composite sample. We can clearly see that for 
luminous sources (with $\mathrm{L}_{[\mathrm{O} \text { III }]}>10^{41} \mathrm{erg} \mathrm{s}^{-1}$ ) in our sample, there is no difference in the [O III] flux distributions between PBL and NPBL Sy2s. For comparison, sources with lower luminosities are also plotted, most of which are much weaker in the [O III] flux. We conclude that most of the nondetections for our luminous sources are likely due to the weakness or lack of PBL but not due to the limited sensitivities of the spectropolarimetric data.

We then verify if our results are biased by combining samples with different selection criteria and survey depths into one single sample, i.e., if some of samples tend to select more obscured sources but with poor spectropolarimetric data, and/or some others tend to select less obscured sources but with better spectropolarimetric data. By plotting in Fig. 6 the "T" ratio vs. [O III] luminosity for sources in each subsample, we can see this is not the case for our composite sample. We find that each subsample spans a similar obscuration range with that of the composite sample, and the difference in the obscuration between PBL and NPBL Sy2s are also visible in most of the subsamples.

We made an additional test to check whether the distributions of $z$ and [O III] luminosity for our luminous sample are different (see Fig. 1 and 2 (a)). Using ASURV, we get average values of $\langle z\rangle=0.02 \pm 0.003$ and $\left.\left\langle\log \mathrm{L}_{[\mathrm{O}} \mathrm{III}\right]\right\rangle=42.198 \pm 0.121$ for PBL Sy2s whereas $\langle z\rangle$ $=0.017 \pm 0.004$ and $\left\langle\log \mathrm{L}_{[\mathrm{O} \text { III }]}\right\rangle=41.991 \pm 0.157$ for NPBL Sy2s. The distributions of $z$ and $[\mathrm{O} \mathrm{III}]$ luminosity for luminous sources are similar at levels of $\mathrm{p}_{\text {null }}=29.4 \%$ and $\mathrm{p}_{\text {null }}$ $=30.6 \%$, respectively. The similarity indicates that the difference in absorption could not be biased by different redshift/luminosity which both affect the visibility of PBL. Also, the dilution effect, which is dependent of the redshift and luminosity, might bias the visibility of PBL for less luminous Sy2s, but itself alone can not explain the difference in the absorption between PBL and NPBL Sy2s. Actually we note that the dilution effect to the visibility of PBL is much weaker for our luminous sources. This can be seen from the fact that PBL Sy2s can be detected in most of luminous Sy2s with smaller obscuration $\left(\mathrm{N}_{\mathrm{H}}<10^{23.8} \mathrm{~cm}^{-2}\right.$ or " $\mathrm{T}$ " ratio $>10^{-0.7}$ ). The dilution effect in X-ray (to the measurement of $\mathrm{N}_{H}$ ) is also much weaker for luminous Sy2s. We conclude that there is no observational bias which can produce the difference in absorption between two types of Sy2s in our sample, and a physical link between the visibility of PBL and nuclear obscuration is required.

\section{2. $\quad$ physical explanation to the difference in absorption}

The results presented here for luminous Sy2s can be interpreted within the context of the unified model for Seyfert galaxies, in agreement with the torus geometry portraited by Heisler et al. (1997): the main electron scattering is confined to a conical region that is close to the thickness of the torus. More inclined Sy2s could have the broad line scattering screen 
also obscured thus make PBL weaker or nondetectable. According to the unified model, more inclined sources expect heavier obscuration, thus explain the difference in obscuration between PBL and NPBL Sy2s.

Note the high detection rate $(86 \%)$ of PBL in luminous Sy2s with $\mathrm{N}_{H}<10^{23.8} \mathrm{~cm}^{-2}$ suggests that in most sources the Compton-thin X-ray obscuring material can not have much larger scale than the scattering screen, supporting the torus scheme of the unified model. Furthermore, while extended obscuration from the host galaxy might explain the absence of PBL in some sources (such as NGC 5506, see Lumsden et al. 2004), it could not be the major cause, otherwise we should have seen a large number of NPBL Sy2s among luminous Sy2s with $\mathrm{N}_{H}<10^{23.8} \mathrm{~cm}^{-2}$. It's interesting to note that NGC 5506 is one of two intermediate Seyferts without PBL detected (the other one is Mrk 334, see §2), both with the BLR visible in near infrared. This suggests extended obscuration from the host galaxy is also a plausible cause in the absence of PBL in Mrk 334.

We also note that while using the "T" ratio as indicator of nuclear obscuration, the difference between two types of Sy2s becomes more significant (see Fig. 2 (d)). We point out that this is mainly because for Compton thick sources, we can only give lower limits of $\sim 10^{24} \mathrm{~cm}^{-2}$ for $\mathrm{N}_{\mathrm{H}}$, but the "T" ratio is a continuous variable as long as they are detected in the X-ray band. Fig. 7 shows the observed 2-10 keV X-ray flux against the extinctioncorrected [O III] flux for Compton-thick Sy2s (with a lower limit of $10^{24} \mathrm{~cm}^{-2}$ of $\mathrm{N}_{\mathrm{H}}, 11$ PBL and 10 NPBL Sy2s). Interestingly, we find although the lower limits of $\mathrm{N}_{\mathrm{H}}$ are the same for two types of Compton thick Sy2s, NPBL Sy2s in the figure tend to be weaker in X-ray (with a confidence level of 97.6\%). We note that two types of Compton-thick Sy2s have similar large FeK line EW $(>1 \mathrm{keV})$ suggesting the X-ray spectra in both types are reflection dominated. In this case, smaller "T" ratio in NPBL Sy2s can also be explained by higher inclination: sources viewed at higher inclination could have a large fraction of inner surface of the torus, where the reflection component is produced, blocked from our line of sight, thus expect weaker X-ray emission.

This work was supported by Chinese NSF through NSF10473009/NSF10533050, and the CAS "Bai Ren" project at University of Science and Technology of China. The work in this paper has made use of the NASA's Astrophysics Data System Abstract Service and the NASA/IPAC Extragalactic Database (NED), which is operated by the Jet Propulsion Laboratory, California Institute of Technology, under contract with NASA. This study has also made use of the HEASARC on-line data archive services, supported by NASA GSFC. 


\section{A. Notes on Individual Objects}

In this section, we present brief discussions for the X-ray data of 3 sources in the sample. All the errors quoted are at the $90 \%$ level of confidence.

NGC 34: we model the $X M M-$ Newton $0.5-8.0 \mathrm{keV}$ spectrum of this source with pexrav model $(\mathrm{C} /$ dof $=362 / 448)$ in terms of its low "T" ratio of $0.03(<0.1)$. However, the Fe $\mathrm{K}$ line is marginally detected with a upper limit EW of $321 \mathrm{eV}$. We then fit the spectrum with an absorbed powerlaw model which gives $\Gamma=1.68_{-0.17}^{+0.11}$ but no intrinsic absorption, plus Fe K line with $\mathrm{EW}=386(<1047) \mathrm{eV}$. The model yields a worse fit with $\mathrm{C} / \mathrm{dof}=389 / 448$. We note the steep spectrum slope may attribute to the host galaxy thermal emission in the soft X-ray band. From the lower "T" ratio of $0.03(<0.1)$ and better C statistic of pexrav model, we consider the galaxy as Compton-thick and give a lower limit of $10^{24} \mathrm{~cm}^{-2}$ to $\mathrm{N}_{\mathrm{H}}$.

NGC 5728: The Chandra $0.5-8 \mathrm{keV}$ spectrum is parameterized here with an absorbed powerlaw $\left(\Gamma=2.73 \pm 0.17, \mathrm{~N}_{\mathrm{H}}=7.8_{-1.4}^{+1.5} \times 10^{23} \mathrm{~cm}^{2}\right)$ plus a $0.4 \%$ scattered component. The Fe $\mathrm{K}$ line is detected at $6.4 \mathrm{keV}$ with $\mathrm{EW}=1100_{-270}^{+320} \mathrm{eV}$. For the lower "T" ratio and large Fe line EW, we then fit the spectrum of this galaxy with pexrav model plus Gaussian line. However, the fitting is unacceptable $(\mathrm{C} / \mathrm{dof}=710 / 300)$. So we do not regard it as a Compton-thick one in this paper. We note considering it as Compton-thick does not affect our results presented here.

NGC 6552: We fit the XMM-Newton $0.5-8 \mathrm{keV}$ spectrum of this source by an absorbed powerlaw $\left(\Gamma=2.8_{-0.13}^{+0.37}\right)$ with a $0.75 \%$ scattered component. The best fit $(\mathrm{C} / \mathrm{dof}=$ $121 / 166)$ gives $\mathrm{N}_{\mathrm{H}}=7.1_{-1.0}^{+4.0} \times 10^{23} \mathrm{~cm}^{2}$. The Fe $\mathrm{K}$ line is detected at $6.4 \mathrm{keV}$ with $\mathrm{EW}=$ $1408_{-883}^{+668} \mathrm{eV}$. However, the pexrav model can also give an acceptable fitting of the spectrum with $\Gamma=2.86_{-0.47}^{+0.34}$ and Fe K line $\mathrm{EW}=4990_{-2390}^{+3910}(\mathrm{C} / \mathrm{dof}=128 / 166)$. The fitted $2-10 \mathrm{keV}$ flux is $2.32 \times 10^{-13} \mathrm{erg} \mathrm{s}^{-1} \mathrm{~cm}^{-2}$. We adopt the absorbed powerlaw model for the spectrum fitting in the paper in terms of the better $\mathrm{C}$ statistic. We note that the consideration of it as Compton thick will not affect our results presented in the paper.

\section{REFERENCES}

Acker, A., Stenholm, B., \& Veron, P. 1991, A\&AS, 87, 499

Alexander, D. M. 2001, MNRAS, 320, L15

Alexander, D. M., Heisler, C. A., Young, S., et al. 2000, MNRAS, 313, 815

Antonucci, R., \& Miller, J. S. 1985, ApJ, 297, 621 
Antonucci, R. 1993, ARA\&A, 31, 473

Awaki, H., Ueno, S., Taniguchi, Y., \& Weaver, K. A. 2000, ApJ, 542, 175

Bassani, L., Dadina, M., Maiolino, R., Salvati, M., Risaliti, G., della Ceca, R., Matt, G., \& Zamorani, G. 1999, ApJS, 121, 473

Bianchi, S., Balestra, I., Matt, G., et al. 2003, A\&A, 402, 141

Bianchi, S., Miniutti, G., Fabian, A., \& Iwasawa, K. 2005a, MNRAS, 360, 380

Bianchi, S., Guainaazi, M., Matt, G., Chiaberge, M., Iwasawa, K., Fiore, F., \& Maiolino, R. 2005b, A\&A, 442, 185

Braito, V., Framcescjomo, A., Della Ceca,R., et al. 2003, A\&A, 398, 107

Cash. W. 1979, ApJ, 228, 939

Cappi, M., Panessa, F., Bassani, L., et al. 2006, A\&A, 446, 459

Cheng, L. P., Zhao, Y. H., \& Wei, J. Y. 2002, CHJAA, 2, 408

Colina L., Sparks W. B., \& Macchetto F., 1991, ApJ, 370, 102

Cruz-Gonzalez, I., et al. 1994, ApJS, 94, 47

Dadina, M., \& Cappi, M. 2004, A\&A, 413, 921

de Grijp, M. H. K., Keel, W. C., Miley, G. K., \& Lub, J. 1992, A\&AS, 96, 389

Dahari, O., \& De Robertis, M. M. 1988, ApJS, 67, 249

de Robertis, M. M., \& Osterbrock. D. E., 1986, ApJ, 301, 98

Dewangan, G. C., Griffiths, R. E., \& Schurch, N. J. 2003, ApJ, 592, 52

Dewangan, G. C., \& Griffiths, R. E., 2005, ApJ, 625, L31

Duc, P. A., Mirabel, I. F., \& Maza, J. 1997, A\&AS, 124, 533

Feigelson, E. D., \& Nelson, P. I. 1985, ApJ, 293, 192

Franceschini, A., et al. 2003, MNRAS, 343, 1181

Fraquelli, H. A., Storchi-Bergmann, T., \& Levenson, N. A. 2003, MNRAS, 341, 449

Georgantopoulos, I., \& Zezas, A. 2003, ApJ, 594, 704 
Guainazzi, M., Matt, G., Antonelli, L. A., Fiore, F., Piro, L. \& Ueno, S. 1998, MNRAS, 298, 842

Guainazzi, M., Rodriguez-Pascual. P., Fabian, A. C., Iwasawa, K., \& Matt, G. 2004, MNRAS, 355, 297

Guainazzi, M., Fabian, A. C., Iwasawa, K., Matt, G., Fiore, F. 2005a, MNRAS, 356, 295

Guainazzi, M., Matt, G., \& Perola, G. C. 2005b, A\&A, 444, 119

Gu, Q., Maiolino, R., \& Dultzin-Hacyan, D. 2001, A\&A, 366, 765

Gu, Q., \& Huang, J. 2002, ApJ, 579, 205

Heisler, C. A., Lumsden, S. L., \& Bailey, J. A. 1997, Nature, 385, 700

Ho, L., Filippenko, V., \& Sargent, W. L. W., 1997, ApJS, 112, 315

Inglis, M. D., et al. 1993, MNRAS, 263, 895

Imanishi, M., \& Terashima, Y. 2004, AJ, 127, 758

Iwasawa, K., Lee, J. C., \& Young, A. J. et al. 2004, MNRAS, 347, 4111

Kay, L. E., \& Moran, E. C., 1998, PASP, 110, 1003

ollatschny, W., Fricke, K. J., Biermann, P., et al. 1983, A\&A, 119, 80

Lonsdale, C. J., 1992, ApJ, 391, 629

Lumsdem, S. L., Heisler, C. A., \& Bailey, J. A., Hough, J. H., Young, S. 2001, MNRAS, 327, 459

Lumsdem, S. L. \& Alexander, D. M. 2001, MNRAS, 328, L32

Lumsden, S., Alexander, D., \& Hough, J., 2004, MNRAS, 348, 1451

Levenson, N. A., Weaver, K. A., Heckman, T. M., Awaki, H., \& Terashima, Y. 2005, ApJ, 618,167

Levenson, N. A., Heckman, T. M., Krolik, J. H., et al. 2006, astro-ph/0605438

Maiolino, R., Salvati, M., Bassani, L., Dadina, M., della Ceca, R., Matt, G., Risaliti, G., \& Zamorani, G. 1998, A\&A, 338, 781

Magdziarz, P., \& Zdziarshi, A. A. 1995, MNRAS, 273, 837 
Masanori, O., Yasushi, F., \& Naoko, I. 2004, PASJ, 56, 425

Matsumoto, C., Nava, A., Maddox, L. A., Leighly, K. M., Grupe, D., Awaki, H., \& Ueno, S. 2004, ApJ, 617, 930

Matt, G., 2000a, A\&A, 355, L31

Matt, G., Fabian, A. C., Guainazzi, M., et al. 2000b, MNRAS, 318, 173

Matt, G., Bianchi, S., Guainazzi, M., Brandt, W. N., Fabian, A. C., Iwasawa, K., \& Perola, G. C. 2003, A\&A, 339, 519

Matt, G., Bianchi, S., D’Ammando, F., \& Martocchia, A. 2004, A\&A, 421, 473

Miller, J. S., \& Goodrich, R. W., 1990, ApJ, 355, 456

Moran, E. C., Halpern, J. P., Bothun, G. D., \& Becker, R. H. 1992, AJ, 104, 990

Moran, E. C., Barth, A. J., Kay, L. E., \& Filippenko, A. V. 2000, ApJ, 540, L73

Moran, E. C., Kay, L. E., Davis, M., Filippenko, A. V., \& Barth, A. J. 2001, ApJ, 556, L75

Mulchaey, J., Koratkar, A., Ward, M. J., et al. 1994, ApJ, 436, 586

Murayama, T., Taniguchi, Y., \& Iwasawa, K. 1998, AJ, 115, 460

Nicastro, F. Martocchia, A. \& Matt, G. 2003, ApJ, 589, L13

Oliva, E., Salvati, M., Moorwood, A. F. M., \& Marconi, A. 1994, A\&A, 288, 457

Panessa, F., \& Bassani, L. 2002, A\&A, 394, 435

Polletta, M., Bassani, L., Malaguti, G., Palumbo, G. G. C., \& Caroli, E. 1996, ApJS, 106, 399

Prieto, M. Almudena, Perez Garcia, A. M., \& Rodriguez Espinosa, J. M. 2002, MNRAS, 329, 309

Pernechele, C., Berta, S., Marconi, A. 2003, MNRAS, 338, 13

Risaliti, G., Gilli, R., Maiolino, R., Salvati, M., 2000, A\&A, 357, 13

Risaliti, G. 2002, A\&A, 386, 379

Smith, D. A., \& Wilson, A. S., 2001, ApJ, 557, 180 
Storchi-Bergmann, T., \& Pastoriza, M. G., 1989, ApJ, 347, 195

Storchi-Bergmann, T., Wilson, A. S., Baldwin, J. A. 1992, ApJ, 396, 45

Storchi-Bergmann, T., Kinney, A. L., \& Challis, P. 1995, ApJS, 98, 103

Terlevich, R., Melnick, J., Masegosa, J., Moles, M., \& Copetti, M., 1991, A\&AS, 91, 285

Turner, T. J., George, I. M., Nandra, K., Mushotzky, \& R. F. 1997, ApJ, 488, 164

Tran, H. D., Miller, J. S., \& Kay, L. E., 1992, ApJ, 397, 452

Tran, H. D., 1995, ApJ, 440, 565

Tran, H. D., 2001, ApJ, 554, L19

Tran, H. D., 2003, ApJ, 583, 632

Ueno, S., Ward, M. J., O’Brien, P. T., et al. 2000, AdSpR, 25, 823

Vaceli, M. S., Viegas, S. M., Gruenwald, R., \& Souza, R. E. 1997, AJ, 114, 1345

Veilleux, S., Kim, D. C., Sanders, D., Mazzarella, J. M., \& Soifer, B. T., 1995, ApJS, 98, 171

Veilleux, S., Goodrich, R. W., Hill, G. J., 1997, ApJ, 477, 631

Vignali, C., \& Comastri, A. 2002, A\&A, 381, 834

Vignali, C., Alexander, D. M., \& Comastri, A. 2004, MNRAS, 354, 720

Whittle, M., 1992, ApJS, 79, 49

Young, S., Hough, J. H., Efstathiou, A., Wills, B. J., Bailey, J. A., Ward, M. J., \& Axon, D. J. 1996, MNRAS, 281, 1206

Yu, P., \& Hwang, C. 2005, ApJ, 631, 720 


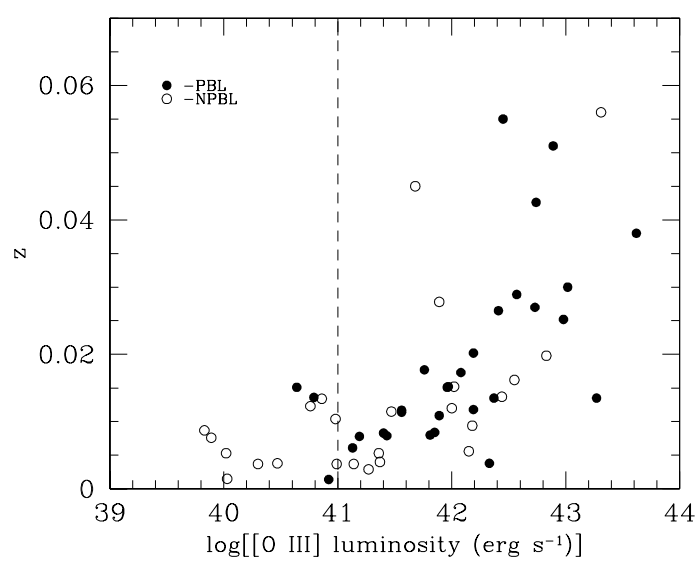

Fig. 1.- Redshift vs. [O III] $\lambda 5007$ luminosity for two types of Sy2s in the sample. solid circles stand for PBL Sy2s and open circles for NPBL Sy2s. 

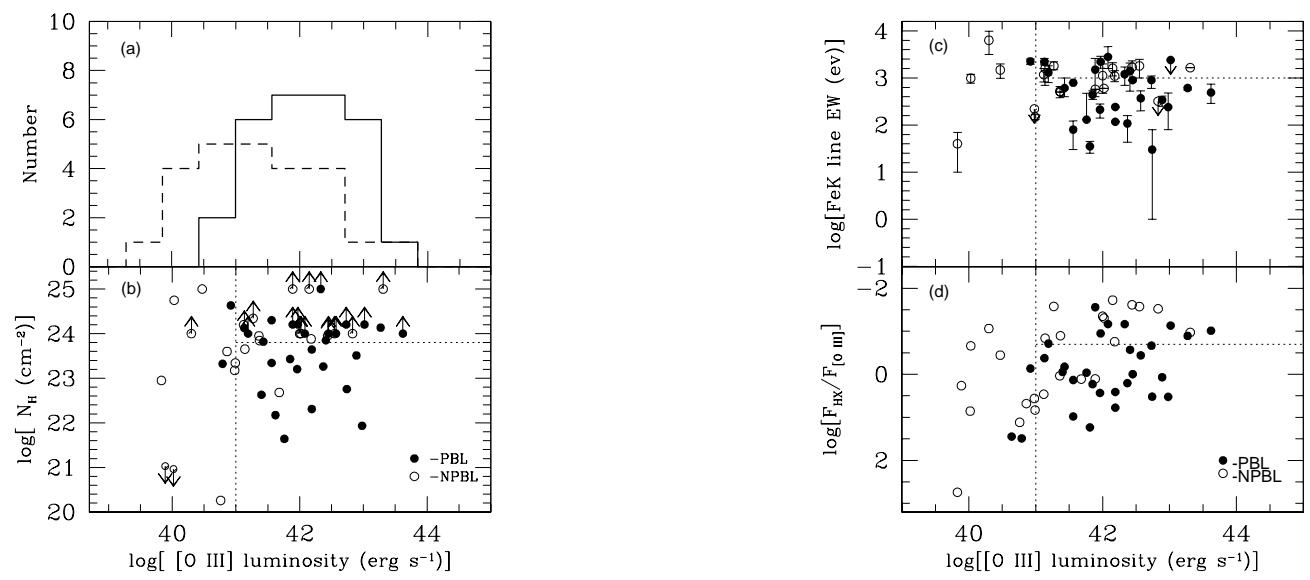

Fig. 2.- The left bottom panel plots the absorption column density $\mathrm{N}_{\mathrm{H}}$ versus the [O III] $\lambda 5007$ luminosity for PBL and NPBL Sy2s. The distribution of the [O III] $\lambda 5007$ luminosity for two types of Sy2s is presented in the left top panel (solid line for PBL Sy2s and dashed line for NPBL Sy2s). The right panel is the plot of FeK line EW (top) and "T" ratio (bottom) versus the [O III] $\lambda 5007$ luminosity. . 

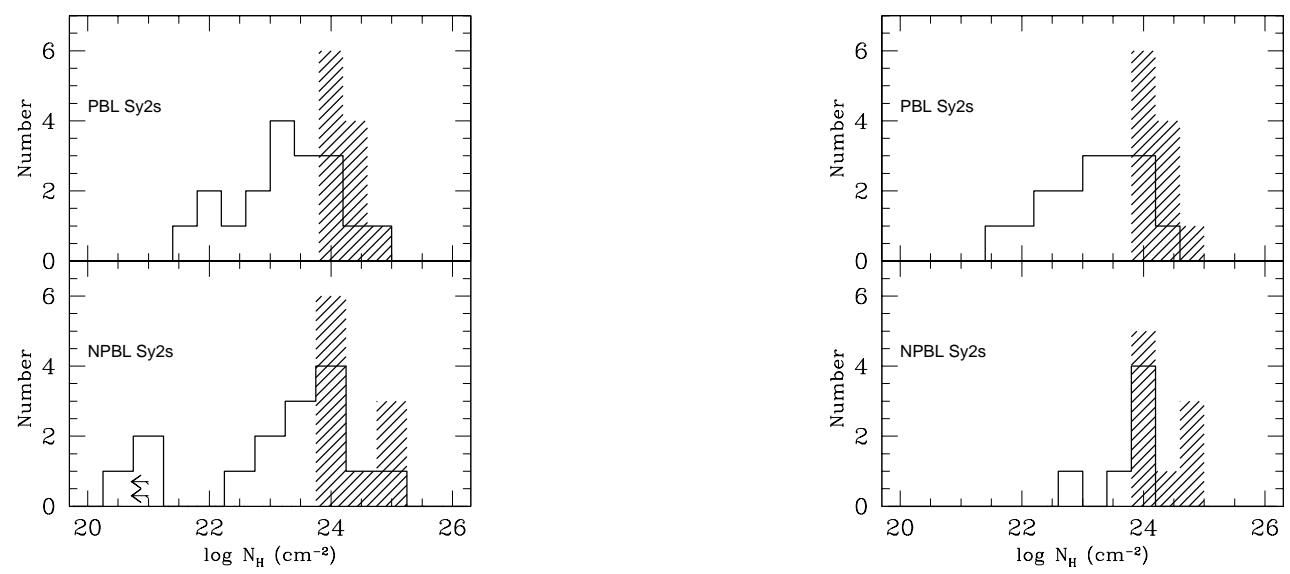

Fig. 3.- $\mathrm{N}_{\mathrm{H}}$ distributions for all the Sy2s in our sample (left) and luminous sources with $\log \mathrm{L}_{[\mathrm{O} \text { III }]}>41 \mathrm{erg} \mathrm{s}^{-1}$ (right). Shaded areas denote lower limits. The arrows denote the upper limits of $\mathrm{N}_{\mathrm{H}}$. 

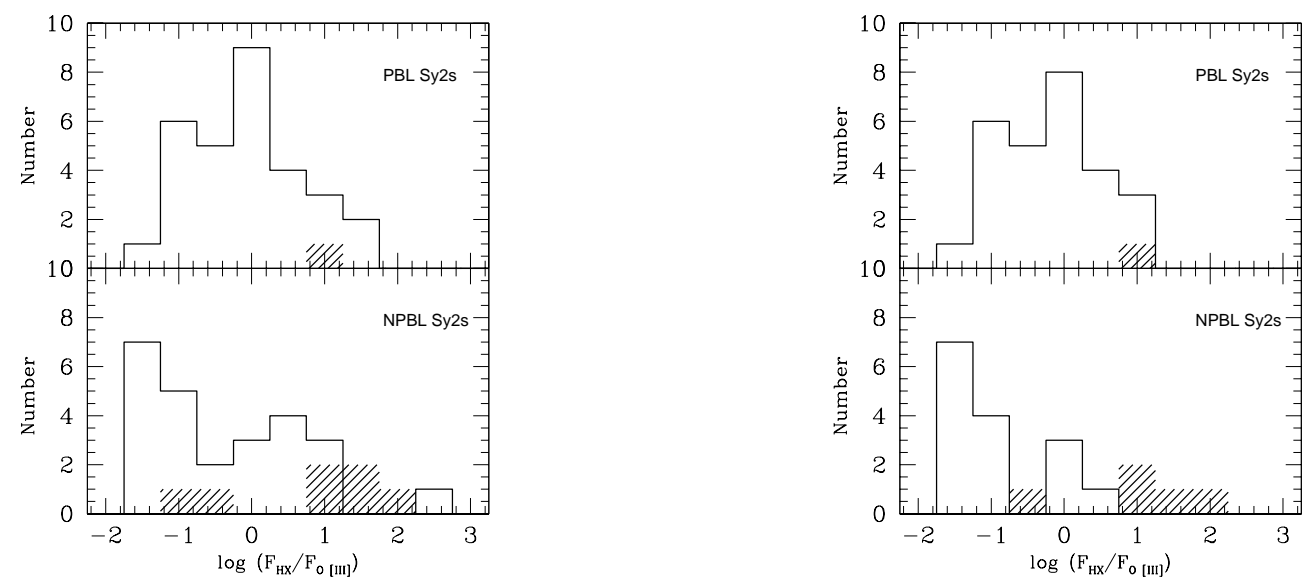

Fig. 4.- Distributions of the "T" ratio for all Sy2s (left) and for luminous Sy2s only (right) in our sample. Shaded areas denote the 8 sources with only hard X-ray upper limits. 


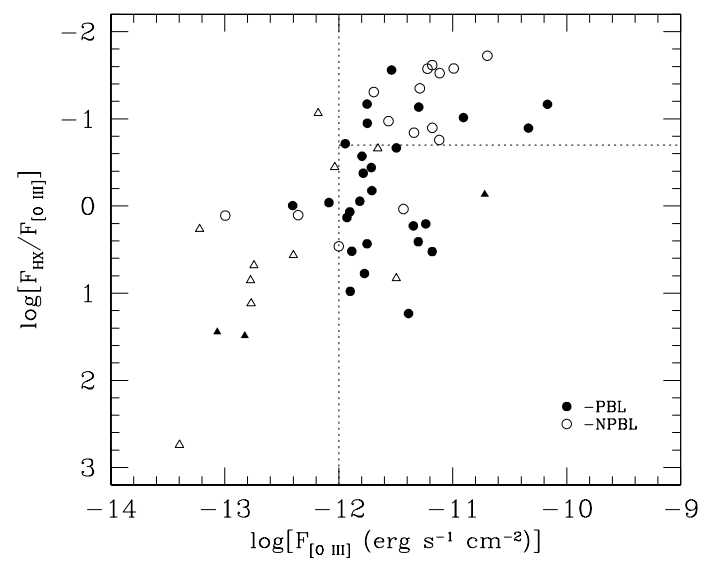

Fig. 5.- The plot of "T" ratio vs. extinction-corrected [O III] flux. Sources with luminosity $\left.\mathrm{L}_{[\mathrm{O}} \mathrm{III}\right]<10^{41} \mathrm{erg} \mathrm{s}^{-1}$ are plotted as triangles. 


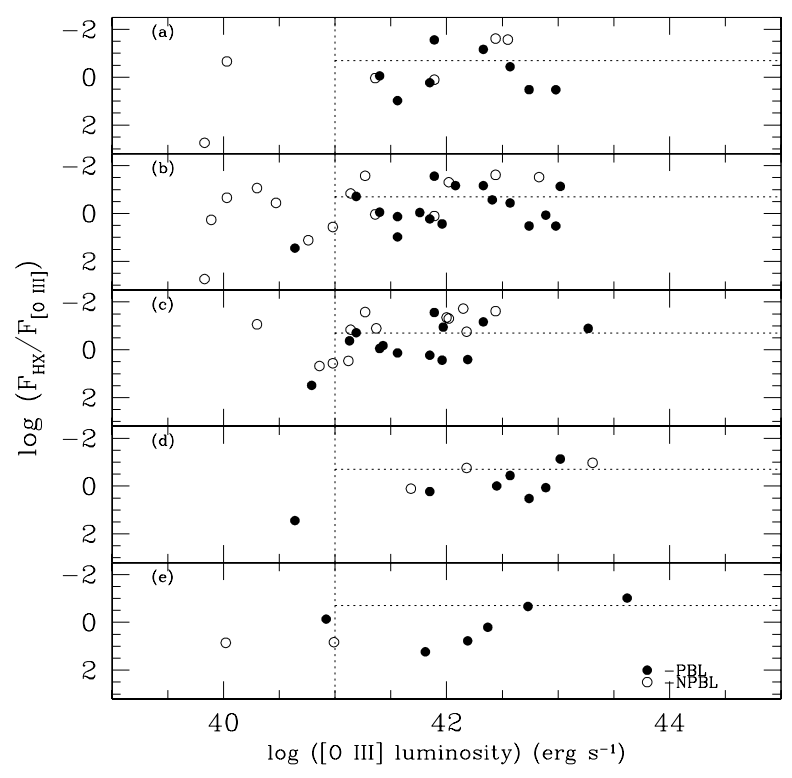

Fig. 6. - The plot of "T" ratio vs. [O III] $\lambda 5007$ luminosity for different spectropolarimetric subsamples. (a): Lumsden et al. (2001)'s sample, (b): Tran (2001)'s sample, (c): Moran et al. (2000)'s sample, (d): Young et al. (1996)'s sample, (e): other surveys. 


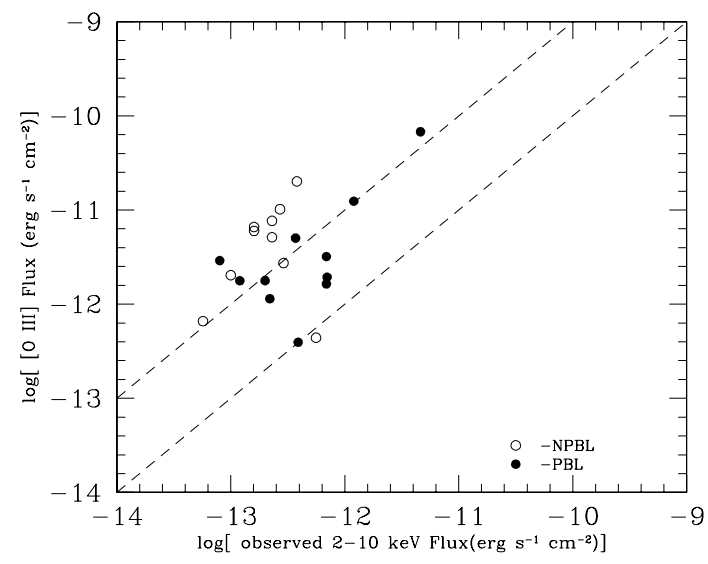

Fig. 7.- The observed [O III] flux (extinction corrected) against X-ray $(2-10 \mathrm{keV})$ flux for Compton-thick Sy2s (with $\mathrm{N}_{\mathrm{H}}>10^{24} \mathrm{~cm}^{-2}$ ). The dashed lines represent $\mathrm{F}_{[\mathrm{O} \text { III }]}=10$ $\mathrm{F}_{2-10 \mathrm{keV}}$ (upper) and $\mathrm{F}_{[\mathrm{O} \mathrm{III}]}=\mathrm{F}_{2-10 \mathrm{keV}}$ (lower). 
Table 1. Optical and Hard X-Ray Data for Seyfert 2 Galaxies with/out PBL

\begin{tabular}{|c|c|c|c|c|c|c|c|c|c|c|}
\hline (1) ${ }^{\text {Name }}$ & $\begin{array}{l}\mathrm{z} \\
(2)\end{array}$ & $\begin{array}{l}\text { PBL? } \\
\text { (3) }\end{array}$ & $\begin{array}{c}\text { References } \\
\text { (4) }\end{array}$ & $\begin{array}{c}\mathrm{F}_{\lambda 5007} \\
(5)\end{array}$ & $\begin{array}{c}\left.\mathrm{L}_{[\mathrm{O}} \mathrm{III}\right] \\
(6)\end{array}$ & $\begin{array}{c}\text { References } \\
(7)\end{array}$ & $\begin{array}{c}\mathrm{F}_{2-10 \mathrm{keV}} \\
(8)\end{array}$ & $\begin{array}{c}\log _{10} \mathrm{~N}_{\mathrm{H}} \\
(9)\end{array}$ & $\begin{array}{c}\mathrm{EW}(\mathrm{Fe}) \\
(10)\end{array}$ & $\begin{array}{c}\text { References } \\
\text { (11) }\end{array}$ \\
\hline \multicolumn{11}{|c|}{ Lumsden et al. (2001)'s sample: } \\
\hline Mrk 334 & 0.022 & $\mathrm{n}$ & 66 & 2.0 & 42.34 & 22 & $<13$ & 20.643 & $\ldots$ & 36 \\
\hline IRAS 00198-7926 & 0.0728 & $\mathrm{n}$ & 2 & 0.36 & 42.67 & 16 & $<0.1$ & $>24$ & $\ldots$ & 62 \\
\hline NGC 1068 & 0.0038 & $\mathrm{y}$ & $3 \mathrm{~L}$ & 67.8 & 42.33 & 22 & 4.62 & $>25$ & $1200 \pm 500$ & 5 \\
\hline NGC 1143 & 0.0291 & $\mathrm{n}$ & 2 & 0.48 & 41.97 & 2 & $\ldots$ & $\ldots$ & $\ldots$ & $\ldots$ \\
\hline IRAS 04259-0440 & 0.0155 & $\mathrm{n}$ & 2 & 1.3 & 41.85 & 2 & $\ldots$ & $\ldots$ & $\ldots$ & $\ldots$ \\
\hline IRAS 05189-2524 & 0.0426 & $\mathrm{y}$ & $1 \mathrm{~A}$ & 1.3 & 42.74 & 2 & 4.3 & 22.756 & $30_{-30}^{+50}$ & 2 \\
\hline NGC 4388 & 0.0084 & $\mathrm{y}$ & $1 \mathrm{~A}$ & 4.51 & 41.85 & 22 & 7.62 & 23.43 & $\begin{array}{r}-30 \\
440_{-90}^{+90}\end{array}$ & 5 \\
\hline IC 3639 & 0.0109 & $\mathrm{y}$ & 2 & 2.9 & 41.89 & 27 & 0.08 & $>24.204$ & $1500_{-1100}^{+1100}$ & 7 \\
\hline MCG -3-34-64 & 0.0165 & $\mathrm{y}$ & $1 \mathrm{~A}$ & 4.0 & 42.39 & 2 & 4.0 & 23.614 & $356_{-143}^{+1860}$ & 67 \\
\hline NGC 5135 & 0.0137 & $\mathrm{n}$ & $2,33 \mathrm{~A}$ & 6.61 & 42.44 & 27 & 0.16 & $>23.954$ & $\begin{array}{r}1700_{-800}^{+600} \\
-60\end{array}$ & 8 \\
\hline NGC 5194 & 0.0015 & $\mathrm{n}$ & $2,15 \mathrm{~L}$ & 2.2 & 40.03 & 31 & 0.48 & 24.748 & $986_{-210}^{+210}$ & 5 \\
\hline NGC 5256 & 0.0278 & $\mathrm{n}$ & $2,15 \mathrm{~L}$ & 0.44 & 41.89 & 22 & 0.56 & $>25$ & 575 & 62 \\
\hline Mrk 1361 & 0.0226 & $\mathrm{n}$ & 2 & 1.8 & 42.32 & 2 & $\ldots$ & $\ldots$ & $\ldots$ & $\ldots$ \\
\hline NGC 5929* & 0.0083 & $\mathrm{y}$ & $42 \mathrm{~K}$ & 1.53 & 41.40 & 2 & 1.35 & 22.629 & $\ldots$ & 9 \\
\hline NGC 5995 & 0.0252 & $\mathrm{y}$ & 2 & 6.6 & 42.98 & 2 & 22 & 21.934 & $240_{-160}^{+240}$ & 2 \\
\hline IRAS $19254-7245^{*}$ & 0.0617 & $\mathrm{y}$ & $69 \mathrm{E}$ & 1.26 & 43.06 & 68 & 0.23 & $>24$ & $2000 \pm 600$ & 70 \\
\hline IC 5063 & 0.0114 & $\mathrm{y}$ & $10 \mathrm{~A}$ & 1.26 & 41.56 & 19 & 12 & 23.342 & $80_{-50}^{+42}$ & 49 \\
\hline NGC 7130 & 0.0162 & $\mathrm{n}$ & 2 & 6.0 & 42.55 & 27 & 0.16 & $>24$ & $1800_{-800}^{+700}$ & 12 \\
\hline NGC 7172 & 0.0087 & $\mathrm{n}$ & 2 & 0.04 & 39.83 & 35 & 22 & 22.95 & $40 \pm 30$ & 13 \\
\hline IC 5298 & 0.0273 & $\mathrm{n}$ & 2 & 1.7 & 42.46 & 2 & $\ldots$ & $\ldots$ & 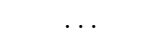 & $\ldots$ \\
\hline NGC 7582 & 0.0053 & $\mathrm{n}$ & $33 \mathrm{~A}, 2$ & 3.69 & 41.36 & 27 & 4.0 & 23.95 & $521_{-141}^{+139}$ & 60 \\
\hline NGC 7674 & 0.0289 & $\mathrm{y}$ & $1 \mathrm{~A}, 20 \mathrm{~L}$ & 1.93 & 42.57 & 22 & 0.7 & $>24$ & $370_{-170}^{+160}$ & 14 \\
\hline \multicolumn{11}{|c|}{ Tran (2001)'s sample: } \\
\hline IRAS 00521-7054 & 0.0689 & $\mathrm{n}$ & $1 \mathrm{~A}$ & 0.36 & 42.62 & 1 & $<31.8$ & $\ldots$ & $\ldots$ & 39 \\
\hline IRAS 01475-0740 & 0.0177 & $\mathrm{y}$ & $15 \mathrm{P}$ & 0.82 & 41.76 & 16 & 0.75 & 21.59 & $130(<344)$ & 13 \\
\hline IRAS 02581-1136 & 0.0299 & $\mathrm{y}$ & $15 \mathrm{~L}$ & 0.07 & 41.16 & 22 & $\ldots$ & $\ldots$ & $\ldots$ & $\ldots$ \\
\hline IRAS 04385-0828 & 0.0151 & $\mathrm{y}$ & $15 \mathrm{LP}$ & 0.086 & 40.64 & 32 & 2.4 & $\ldots$ & $\ldots$ & 39 \\
\hline IRAS 05189-2524 & 0.0426 & $\mathrm{y}$ & $1 \mathrm{~A}$ & 1.3 & 42.74 & 2 & 4.3 & 22.756 & $30_{-30}^{+50}$ & 2 \\
\hline IRAS 15480-0344 & 0.03 & $\mathrm{y}$ & $15 \mathrm{P}, 1 \mathrm{~A}$ & 5.03 & 43.02 & 16 & 0.37 & $>24.204$ & $<2400$ & 7 \\
\hline IRAS $22017+0319$ & 0.0611 & $\mathrm{y}$ & $15 \mathrm{P}, 1 \mathrm{~A}$ & 0.42 & 42.58 & 1 & 3.6 & 22.69 & $380_{-160}^{+180}$ & 18 \\
\hline
\end{tabular}


Table 1-Continued

\begin{tabular}{|c|c|c|c|c|c|c|c|c|c|c|}
\hline $\begin{array}{l}\text { Name } \\
(1)\end{array}$ & $\begin{array}{l}\mathrm{z} \\
(2)\end{array}$ & $\begin{array}{c}\text { PBL? } \\
(3)\end{array}$ & $\begin{array}{l}\text { References } \\
\text { (4) }\end{array}$ & $\begin{array}{c}\mathrm{F}_{\lambda 5007} \\
(5)\end{array}$ & $\begin{array}{c}\mathrm{L}_{[} \mathrm{O} \text { IIII] } \\
(6)\end{array}$ & $\begin{array}{c}\text { References } \\
(7)\end{array}$ & $\begin{array}{c}\mathrm{F}_{2-10 \mathrm{keV}} \\
(8)\end{array}$ & $\begin{array}{c}\log _{10} \mathrm{~N}_{\mathrm{H}} \\
(9)\end{array}$ & $\begin{array}{c}\mathrm{EW}(\mathrm{Fe}) \\
(10)\end{array}$ & $\begin{array}{c}\text { References } \\
\text { (11) }\end{array}$ \\
\hline IC 5063 & 0.0114 & $\mathrm{y}$ & $10 \mathrm{~A}$ & 1.26 & 41.56 & 19 & 12 & 23.342 & $80_{-50}^{+42}$ & 49 \\
\hline MCG -3-34-64 & 0.0165 & $\mathrm{y}$ & $1 \mathrm{~A}$ & 4.0 & 42.39 & 2 & 4.0 & 23.614 & $356_{-143}^{+186}$ & 67 \\
\hline Mrk 348 & 0.0151 & $\mathrm{y}$ & $20 \mathrm{~L}$ & 1.77 & 41.96 & 22 & 4.8 & 23.204 & $212_{-72}^{+68}$ & 21 \\
\hline MCG -3-5-87 & 0.0317 & $\mathrm{y}$ & $15 \mathrm{P}$ & 0.37 & 41.93 & 32 & $\ldots$ & $\ldots$ & $\ldots$ & $\ldots$ \\
\hline Mrk 463E & 0.051 & $\mathrm{y}$ & $20 \mathrm{~L}, 1 \mathrm{~A}$ & 1.25 & 42.89 & 22 & 1.46 & 23.51 & $340_{-90}^{+70}$ & 6 \\
\hline NGC 424 & 0.0117 & $\mathrm{y}$ & $23 \mathrm{C}$ & 1.18 & 41.56 & 24 & 1.6 & 24.301 & 790 & 25 \\
\hline NGC 513 & 0.0195 & $\mathrm{y}$ & $26 \mathrm{~L}$ & 0.16 & 41.14 & 32 & $\ldots$ & $\ldots$ & $\ldots$ & $\ldots$ \\
\hline NGC 1068 & 0.0038 & $\mathrm{y}$ & $3 \mathrm{~L}$ & 67.8 & 42.33 & 22 & 4.62 & $>25$ & $1200 \pm 500$ & 5 \\
\hline NGC 4388 & 0.0084 & $\mathrm{y}$ & $1 \mathrm{~A}$ & 4.51 & 41.85 & 22 & 7.62 & 23.43 & $440_{-90}^{+90}$ & 5 \\
\hline NGC 5506 & 0.0062 & $\mathrm{n}$ & 15 & 3.33 & 41.45 & 58 & 58 & 22.46 & $86_{-10}^{+24}$ & 11 \\
\hline NGC 5995 & 0.0252 & $\mathrm{y}$ & 2 & 6.6 & 42.98 & 2 & 22 & 21.934 & $240_{-160}^{+240}$ & 2 \\
\hline NGC 6552 & 0.0265 & $\mathrm{y}$ & $15 \mathrm{P}$ & 1.6 & 42.41 & 32 & 0.43 & 23.85 & $1408_{-883}^{+668}$ & 13 \\
\hline NGC 7674 & 0.0289 & $\mathrm{y}$ & $1 \mathrm{~A}, 20 \mathrm{~L}$ & 1.93 & 42.57 & 22 & 0.7 & $>24$ & $370_{-170}^{+160}$ & 14 \\
\hline NGC 7682 & 0.0171 & $\mathrm{y}$ & $15 \mathrm{P}$ & 0.87 & 41.76 & 28 & $<13$ & $\ldots$ & (1) & 39 \\
\hline IC 3639 & 0.0109 & $\mathrm{y}$ & 2 & 2.9 & 41.89 & 27 & 0.08 & $>24.204$ & $1500_{-1100}^{+1100}$ & 7 \\
\hline IRAS 00198-7926 & 0.0728 & $\mathrm{n}$ & 2 & 0.36 & 42.67 & 16 & $<0.1$ & $>24$ & $\ldots$ & 62 \\
\hline IRAS 03362-1642 & 0.0372 & $\mathrm{n}$ & $3 \mathrm{~L}$ & 0.13 & 41.62 & 16 & $\ldots$ & $\ldots$ & $\ldots$ & $\ldots$ \\
\hline IRAS $19254-7245^{*}$ & 0.0617 & $\mathrm{y}$ & $69 \mathrm{E}$ & 1.26 & 43.06 & 68 & 0.23 & $>24$ & $2000 \pm 600$ & 70 \\
\hline NGC 5194 & 0.0015 & $\mathrm{n}$ & $2,15 \mathrm{~L}$ & 2.2 & 40.03 & 31 & 0.48 & 24.748 & $986_{-210}^{+210}$ & 5 \\
\hline NGC 5256 & 0.0278 & $\mathrm{n}$ & $2,15 \mathrm{~L}$ & 0.44 & 41.89 & 22 & 0.56 & $>25$ & 575 & 62 \\
\hline Mrk $573^{*}$ & 0.0173 & $\mathrm{y}$ & $33 \mathrm{~S}$ & 1.77 & 42.08 & 22 & 0.12 & $>24$ & $2800_{-1220}^{+1820}$ & 13 \\
\hline NGC 34 & 0.0198 & $\mathrm{n}$ & $15 \mathrm{P}, 33 \mathrm{~A}$ & 7.68 & 42.83 & 34 & 0.23 & $>24$ & $<321$ & 13 \\
\hline NGC 1144 & 0.0289 & $\mathrm{n}$ & $15 \mathrm{P}, 33 \mathrm{~A}$ & 0.39 & 41.87 & 34,35 & $<12$ & 20.699 & $\ldots$ & 36 \\
\hline NGC 1241 & 0.0135 & $\mathrm{n}$ & $15 \mathrm{P}$ & 0.91 & 41.57 & 32,35 & $\ldots$ & $\ldots$ & $\ldots$ & $\ldots$ \\
\hline NGC 1320 & 0.0094 & $\mathrm{n}$ & $15 \mathrm{~L}$ & 0.57 & 41.05 & 37 & $<8.2$ & $\ldots$ & $\ldots$ & 39 \\
\hline NGC 1386 & 0.0029 & $\mathrm{n}$ & 23 & 10.2 & 41.27 & 38 & 0.27 & $>24.342$ & $1800_{-300}^{+400}$ & 8 \\
\hline NGC 1667 & 0.0152 & $\mathrm{n}$ & $15 \mathrm{~L}, 23$ & 2.03 & 42.02 & 27 & 0.1 & $>24$ & 600 & 14 \\
\hline NGC 3079 & 0.0038 & $\mathrm{n}$ & $15 \mathrm{~L}$ & 0.92 & 40.47 & 31 & 0.33 & 25 & $1480_{-500}^{+500}$ & 5 \\
\hline NGC 3362 & 0.0276 & $\mathrm{n}$ & $15 \mathrm{~L}$ & 0.13 & 41.36 & 39 & $<12.6$ & $\ldots$ & .. & 39 \\
\hline NGC 3660 & 0.0123 & $\mathrm{n}$ & $15 \mathrm{~L}$ & 0.17 & 40.76 & 27,61 & 2.22 & 20.26 & & 9 \\
\hline NGC 3982 & 0.0037 & $\mathrm{n}$ & $15 \mathrm{~L}, 23$ & 0.66 & 40.3 & 15,31 & 0.057 & $>24$ & $6310_{-3170}^{+3500}$ & 13 \\
\hline NGC 4501 & 0.0076 & $\mathrm{n}$ & $15 \mathrm{~L}$ & 0.06 & 39.89 & 31 & 0.11 & $<21.03$ & & 5 \\
\hline NGC 4941 & 0.0037 & $\mathrm{n}$ & 23 & 4.57 & 41.14 & 38 & 0.66 & 23.653 & $1600_{-900}^{+700}$ & 40 \\
\hline NGC 5135 & 0.0137 & $\mathrm{n}$ & $2,33 \mathrm{~A}$ & 6.61 & 42.44 & 27 & 0.16 & $>23.954$ & $\begin{array}{r}1700_{-800}^{+600} \\
\end{array}$ & 8 \\
\hline
\end{tabular}


Table 1-Continued

\begin{tabular}{|c|c|c|c|c|c|c|c|c|c|c|}
\hline (1) & $\begin{array}{l}\mathrm{z} \\
(2)\end{array}$ & $\begin{array}{l}\text { PBL? } \\
(3)\end{array}$ & $\begin{array}{c}\text { References } \\
\text { (4) }\end{array}$ & $\begin{array}{c}\mathrm{F}_{\lambda 5007} \\
(5)\end{array}$ & $\begin{array}{c}\mathrm{L}_{[\mathrm{O} \quad \mathrm{III}]} \\
(6)\end{array}$ & $\begin{array}{l}\text { References } \\
\text { (7) }\end{array}$ & $\begin{array}{c}\mathrm{F}_{2-10 \mathrm{keV}} \\
(8)\end{array}$ & $\begin{array}{c}\log _{10} \mathrm{~N}_{\mathrm{H}} \\
(9)\end{array}$ & $\begin{array}{c}\mathrm{EW}(\mathrm{Fe}) \\
(10)\end{array}$ & $\begin{array}{c}\text { References } \\
\text { (11) }\end{array}$ \\
\hline NGC 5283 & 0.0104 & $\mathrm{n}$ & $15 \mathrm{~L}, 23$ & 0.4 & 40.98 & 22 & 1.46 & 23.176 & $<220$ & 7 \\
\hline NGC $5347^{*}$ & 0.0078 & $\mathrm{y}$ & $42 \mathrm{~K}$ & 1.14 & 41.19 & 15 & 0.22 & $>24$ & $1300 \pm 500$ & 63 \\
\hline NGC 5695 & 0.014 & $\mathrm{n}$ & $15 \mathrm{~L}, 23$ & 0.081 & 40.55 & 22 & $<0.01$ & $\ldots$ & $\ldots$ & 39 \\
\hline NGC 5929* & 0.0083 & $\mathrm{y}$ & $42 \mathrm{~K}$ & 1.53 & 41.40 & 2 & 1.35 & 22.629 & $\ldots$ & 9 \\
\hline NGC 6890 & 0.0081 & $\mathrm{n}$ & 23 & 0.5 & 40.86 & 43 & $\ldots$ & $\ldots$ & $\ldots$ & $\ldots$ \\
\hline NGC 7172 & 0.0087 & $\mathrm{n}$ & 2 & 0.04 & 39.83 & 35 & 22 & 22.95 & $40 \pm 30$ & 13 \\
\hline NGC 7582 & 0.0053 & $\mathrm{n}$ & $33 \mathrm{~A}, 2$ & 3.69 & 41.36 & 27 & 4.0 & 23.95 & $521_{-141}^{+139}$ & 60 \\
\hline UGC 6100 & 0.0295 & $\mathrm{n}$ & $15 \mathrm{~L}$ & 0.96 & 42.28 & 28 & $<11.4$ & $\ldots$ & $\ldots$ & 39 \\
\hline \multicolumn{11}{|c|}{ Moran et al. (2000)'s sample: } \\
\hline IC 3639 & 0.0109 & $\mathrm{y}$ & 2 & 2.9 & 41.89 & 27 & 0.08 & $>24.204$ & $1500_{-1100}^{+1100}$ & 7 \\
\hline ESO 428-G014 & 0.0056 & $\mathrm{n}$ & 23 & 20.1 & 42.15 & 44 & 0.38 & $>25$ & $1600 \pm 500$ & 63 \\
\hline MCG +1-27-020 & 0.0117 & $\mathrm{n}$ & 23 & $\ldots$ & $\ldots$ & $\ldots$ & $\ldots$ & $\ldots$ & $\ldots$ & $\ldots$ \\
\hline Mrk 3 & 0.0135 & $\mathrm{y}$ & $20 \mathrm{~L}$ & 46.1 & 43.27 & 43 & 5.9 & 24.134 & $610_{-50}^{+30}$ & 45 \\
\hline Mrk 1066 & 0.012 & $\mathrm{n}$ & $20 \mathrm{~L}$ & 5.14 & 42 & 43 & 0.23 & $>24$ & $1120_{-650}^{+850}$ & 13 \\
\hline Mrk 348 & 0.0151 & $\mathrm{y}$ & $20 \mathrm{~L}$ & 1.77 & 41.96 & 22 & 4.8 & 23.204 & $212_{-72}^{+68}$ & 21 \\
\hline NGC 424 & 0.0117 & $\mathrm{y}$ & $23 \mathrm{C}$ & 1.18 & 41.56 & 24 & 1.6 & 24.301 & 790 & 25 \\
\hline NGC 591 & 0.0152 & $\mathrm{y}$ & $23 \mathrm{~K}$ & 1.78 & 41.97 & 43 & 0.2 & $>24.204$ & $2200_{-600}^{+700}$ & 7 \\
\hline NGC 788 & 0.0136 & $\mathrm{y}$ & $46 \mathrm{~L}$ & 0.15 & 40.79 & 41 & 4.62 & 23.324 & $\ldots$ & 9 \\
\hline NGC 1068 & 0.0038 & $\mathrm{y}$ & $3 \mathrm{~L}$ & 67.8 & 42.33 & 22 & 4.62 & $>25$ & $1200 \pm 500$ & 5 \\
\hline NGC 1358 & 0.0134 & $\mathrm{n}$ & 23 & 0.18 & 40.86 & 43 & 0.86 & 23.6 & $\ldots$ & 47 \\
\hline NGC 1386 & 0.0029 & $\mathrm{n}$ & 23 & 10.2 & 41.27 & 38 & 0.27 & $>24.342$ & $1800_{-300}^{+400}$ & 8 \\
\hline NGC 1667 & 0.0152 & $\mathrm{n}$ & $15 \mathrm{~L}, 23$ & 2.03 & 42.02 & 27 & 0.1 & $>24$ & 600 & 14 \\
\hline NGC 1685 & 0.0152 & $\mathrm{n}$ & 23 & 9.09 & 42.67 & 28 & $<2$ & $\ldots$ & $\ldots$ & 39 \\
\hline NGC 2273 & 0.0061 & $\mathrm{y}$ & $23 \mathrm{~K}$ & 1.64 & 41.13 & 48 & 0.69 & $>24.126$ & $2200_{-300}^{+400}$ & 8 \\
\hline NGC 3081 & 0.0079 & $\mathrm{y}$ & $23 \mathrm{~K}$ & 1.95 & 41.43 & 27 & 1.3 & 23.819 & $610_{-210}^{+390}$ & 49 \\
\hline NGC 3281 & 0.0115 & $\mathrm{n}$ & 23 & 1.0 & 41.47 & 64 & 2.9 & 24.197 & $1180_{-361}^{+400}$ & 50 \\
\hline NGC 3982 & 0.0037 & $\mathrm{n}$ & $15 \mathrm{~L}, 23$ & 0.66 & 40.3 & 15,31 & 0.057 & $>24$ & $6310_{-3170}^{+3500}$ & 13 \\
\hline NGC 4117 & 0.0031 & $\mathrm{n}$ & 23 & $\ldots$ & $\ldots$ & $\ldots$ & $<23.2$ & $\ldots$ & $\ldots$ & 39 \\
\hline NGC 4388 & 0.0084 & $\mathrm{y}$ & $1 \mathrm{~A}$ & 4.51 & 41.85 & 22 & 7.62 & 23.43 & $440_{-90}^{+90}$ & 5 \\
\hline NGC 4507 & 0.0118 & $\mathrm{y}$ & $23 \mathrm{~K}$ & 4.98 & 42.19 & 43 & 12.8 & 23.643 & 117 & 51 \\
\hline NGC 4941 & 0.0037 & $\mathrm{n}$ & 23 & 4.57 & 41.14 & 38 & 0.66 & 23.653 & $1600_{-900}^{+700}$ & 40 \\
\hline NGC 5135 & 0.0137 & $\mathrm{n}$ & $2,33 \mathrm{~A}$ & 6.61 & 42.44 & 27 & 0.16 & $>23.954$ & $1700_{-800}^{+600}$ & 8 \\
\hline
\end{tabular}


Table 1-Continued

\begin{tabular}{|c|c|c|c|c|c|c|c|c|c|c|}
\hline (1) ${ }^{\text {Name }}$ & $\begin{array}{l}\mathrm{z} \\
(2)\end{array}$ & $\begin{array}{c}\text { PBL? } \\
\text { (3) }\end{array}$ & $\begin{array}{c}\text { References } \\
\text { (4) }\end{array}$ & $\begin{array}{c}\mathrm{F}_{\lambda 5007} \\
\quad(5)\end{array}$ & $\begin{array}{c}\mathrm{L}_{[\mathrm{O} \quad \mathrm{III}]} \\
(6)\end{array}$ & $\begin{array}{c}\text { References } \\
\text { (7) }\end{array}$ & $\begin{array}{c}\mathrm{F}_{2-10 \mathrm{keV}} \\
(8)\end{array}$ & $\begin{array}{c}\log _{10} \mathrm{~N}_{\mathrm{H}} \\
(9)\end{array}$ & $\begin{array}{c}\mathrm{EW}(\mathrm{Fe}) \\
(10)\end{array}$ & $\begin{array}{c}\text { References } \\
\text { (11) }\end{array}$ \\
\hline NGC 5283 & 0.0104 & $\mathrm{n}$ & $15 \mathrm{~L}, 23$ & 0.4 & 40.98 & 22 & 1.46 & 23.176 & $<220$ & 7 \\
\hline NGC 5728 & 0.0094 & $\mathrm{n}$ & $23,1 \mathrm{~A}$ & 7.61 & 42.18 & 27 & 1.33 & 23.89 & $1100_{-270}^{+320}$ & 13 \\
\hline NGC 5643 & 0.004 & $\mathrm{n}$ & 23 & 6.62 & 41.37 & 43 & 0.84 & 23.845 & 500 & 52 \\
\hline NGC $5347^{*}$ & 0.0078 & $\mathrm{y}$ & $42 \mathrm{~K}$ & 1.14 & 41.19 & 15 & 0.22 & $>24$ & $1300 \pm 500$ & 63 \\
\hline NGC 5695 & 0.014 & $\mathrm{n}$ & $15 \mathrm{~L}, 23$ & 0.081 & 40.55 & 22 & $<0.01$ & $\ldots$ & $\ldots$ & 39 \\
\hline NGC 5929* & 0.0083 & $\mathrm{y}$ & $42 \mathrm{~K}$ & 1.53 & 41.40 & 2 & 1.35 & 22.629 & $\ldots$ & 9 \\
\hline NGC 6890 & 0.0081 & $\mathrm{n}$ & 23 & 0.5 & 40.86 & 43 & $\ldots$ & $\ldots$ & $\ldots$ & $\ldots$ \\
\hline NGC 7672 & 0.0134 & $\mathrm{n}$ & $20 \mathrm{~L}$ & $\ldots$ & $\ldots$ & $\cdots$ & 28.6 & $\ldots$ & $\ldots$ & 39 \\
\hline \multicolumn{11}{|c|}{ Young et al. (1996)'s sample: } \\
\hline IRAS 00521-7054 & 0.0689 & $\mathrm{n}$ & $1 \mathrm{~A}$ & 0.36 & 42.62 & 1 & $<31.8$ & $\cdots$ & $\ldots$ & 39 \\
\hline IRAS 04103-2838 & 0.118 & $\mathrm{n}$ & $1 \mathrm{~A}$ & $\ldots$ & $\ldots$ & $\ldots$ & $\ldots$ & $\ldots$ & $\ldots$ & $\ldots$ \\
\hline IRAS $04210+0400$ & 0.046 & $\mathrm{n}$ & $1 \mathrm{~A}$ & 0.554 & 42.44 & 1 & $\ldots$ & $\ldots$ & $\ldots$ & $\ldots$ \\
\hline IRAS 04229-2528 & 0.044 & $\mathrm{n}$ & $1 \mathrm{~A}$ & 0.216 & 41.99 & 1 & $\ldots$ & $\ldots$ & $\ldots$ & $\ldots$ \\
\hline IRAS 04385-0828 & 0.0151 & $\mathrm{y}$ & $15 \mathrm{LP}$ & 0.086 & 40.64 & 32 & 2.4 & $\ldots$ & $\ldots$ & 39 \\
\hline IRAS 05189-2524 & 0.0426 & $\mathrm{y}$ & $1 \mathrm{~A}$ & 1.3 & 42.74 & 2 & 4.3 & 22.756 & $30_{-30}^{+50}$ & 2 \\
\hline IRAS 11058-1131 & 0.055 & $\mathrm{y}$ & $1 \mathrm{~A}$ & 0.394 & 42.45 & 1 & 0.39 & $>24$ & 900 & 18 \\
\hline MCG -3-34-64 & 0.0165 & $\mathrm{y}$ & $1 \mathrm{~A}$ & 4.0 & 42.39 & 2 & 4.0 & 23.614 & $356_{-143}^{+186}$ & 67 \\
\hline IRAS 08277-0242 & 0.041 & $\mathrm{n}$ & $1 \mathrm{~A}$ & 1.42 & 42.75 & 16 & $\ldots$ & $\ldots$ & $\ldots$ & $\ldots$ \\
\hline IRAS $13452-4155$ & 0.039 & $\mathrm{n}$ & $1 \mathrm{~A}$ & $\ldots$ & $\ldots$ & $\ldots$ & $\ldots$ & $\ldots$ & $\ldots$ & $\ldots$ \\
\hline ESO 273-IG04 & 0.039 & $\mathrm{y}$ & $1 \mathrm{~A}$ & 0.85 & 42.48 & 1 & $\ldots$ & $\ldots$ & $\ldots$ & $\ldots$ \\
\hline IRAS 15480-0344 & 0.03 & $\mathrm{y}$ & $15 \mathrm{P}, 1 \mathrm{~A}$ & 5.03 & 43.02 & 16 & 0.37 & $>24.204$ & $<2400$ & 7 \\
\hline IRAS $20210+1121$ & 0.056 & $\mathrm{n}$ & $1 \mathrm{~A}$ & 2.73 & 43.31 & 1 & 0.29 & $>25$ & 1650 & 18 \\
\hline IRAS $20460+1925$ & 0.181 & $\mathrm{y}$ & $1 \mathrm{~A}$ & 0.112 & 43.02 & 1 & 1.5 & 22.398 & $260_{-137}^{+145}$ & 49 \\
\hline IRAS $22017+0319$ & 0.0611 & $\mathrm{y}$ & $15 \mathrm{P}, 1 \mathrm{~A}$ & 0.42 & 42.58 & 1 & 3.6 & 22.69 & $380_{-160}^{+180}$ & 18 \\
\hline IRAS 23128-5919 & 0.045 & $\mathrm{n}$ & $1 \mathrm{~A}$ & 0.101 & 41.68 & 1 & 0.13 & 22.681 & $\ldots$ & 53 \\
\hline NGC 5506 & 0.0062 & $\mathrm{n}$ & 15 & 3.33 & 41.45 & 58 & 58 & 22.46 & $86_{-10}^{+24}$ & 11 \\
\hline Mrk 463E & 0.051 & $\mathrm{y}$ & $20 \mathrm{~L}, 1 \mathrm{~A}$ & 1.25 & 42.89 & 22 & 1.46 & 23.51 & $340_{-90}^{+70}$ & 6 \\
\hline NGC 4388 & 0.0084 & $\mathrm{y}$ & $1 \mathrm{~A}$ & 4.51 & 41.85 & 22 & 7.62 & 23.43 & $440_{-90}^{+90}$ & 5 \\
\hline NGC 5252 & 0.023 & $\mathrm{y}$ & $1 \mathrm{~A}$ & 0.921 & 42.05 & 1 & 10.7 & 22.461 & $44 \pm 28$ & 13 \\
\hline NGC 5728 & 0.0094 & $\mathrm{n}$ & $23,1 \mathrm{~A}$ & 7.61 & 42.18 & 27 & 1.33 & 23.89 & $1100_{-270}^{+320}$ & 13 \\
\hline NGC 7496 & 0.005 & $\mathrm{n}$ & $1 \mathrm{~A}$ & 0.3 & 40.22 & 1 & $<8$ & 22.699 & & 39 \\
\hline NGC 7674 & 0.0289 & $\mathrm{y}$ & $1 \mathrm{~A}, 20 \mathrm{~L}$ & 1.93 & 42.57 & 22 & 0.7 & $>24$ & $370_{-170}^{+160}$ & 14 \\
\hline
\end{tabular}


Table 1 - Continued

\begin{tabular}{|c|c|c|c|c|c|c|c|c|c|c|}
\hline $\begin{array}{c}\text { Name } \\
(1)\end{array}$ & $\begin{array}{l}\mathrm{z} \\
(2)\end{array}$ & $\begin{array}{c}\text { PBL? } \\
\text { (3) }\end{array}$ & $\begin{array}{c}\text { References } \\
\text { (4) }\end{array}$ & $\begin{array}{c}\mathrm{F}_{\lambda 5007} \\
(5)\end{array}$ & $\begin{array}{c}\mathrm{L}_{[\mathrm{O} \quad \mathrm{III}]} \\
(6)\end{array}$ & $\begin{array}{c}\text { References } \\
(7)\end{array}$ & $\begin{array}{c}\mathrm{F}_{2-10 \mathrm{keV}} \\
(8)\end{array}$ & $\begin{array}{c}\log _{10} \mathrm{~N}_{\mathrm{H}} \\
(9)\end{array}$ & $\begin{array}{c}\mathrm{EW}(\mathrm{Fe}) \\
(10)\end{array}$ & $\begin{array}{c}\text { References } \\
\text { (11) }\end{array}$ \\
\hline \multicolumn{11}{|l|}{ Other surveys: } \\
\hline Mrk 1210 & 0.0135 & $\mathrm{y}$ & $54 \mathrm{~L}$ & 5.8 & 42.37 & 55 & 9.3 & 23.263 & $108_{-65}^{+50}$ & 56 \\
\hline IRAS 18325-5926 & 0.0202 & $\mathrm{y}$ & $58 \mathrm{~A}$ & 1.68 & 42.19 & 58 & 10 & 22.31 & 242 & 30 \\
\hline MCG -5-23-16 & 0.008 & $\mathrm{y}$ & $58 \mathrm{~A}$ & 4.09 & 41.81 & 49 & 70 & 22.25 & $35.2_{-10}^{+9.6}$ & 59 \\
\hline Circinus & 0.0014 & $\mathrm{y}$ & 29 & 19.1 & 40.92 & 17 & 14 & 24.633 & $2250_{-300}^{+260}$ & 4 \\
\hline Mrk 477 & 0.038 & $\mathrm{y}$ & $54 \mathrm{~L}$ & 12.4 & 43.62 & 22 & 1.2 & $>24$ & $490_{-200}^{+250}$ & 49 \\
\hline NGC 2992 & 0.0077 & $\mathrm{y}$ & $58 \mathrm{~A}$ & 1.49 & 41.3 & 58 & 4.5 & 21.84 & $514 \pm 190$ & 49 \\
\hline NGC 7314 & 0.0047 & $\mathrm{y}$ & $58 \mathrm{~A}$ & 17.7 & 42.41 & 49 & 41.2 & 22.02 & $147_{-109}^{+128}$ & 60 \\
\hline NGC 6300 & 0.0037 & $\mathrm{n}$ & $58 \mathrm{~A}$ & 3.2 & 40.99 & 58 & 21.6 & 23.342 & $148_{-18}^{+18}$ & 65 \\
\hline NGC 7212 & 0.027 & $\mathrm{y}$ & $54 \mathrm{~L}$ & 3.2 & 42.73 & 43 & 0.69 & $>24.204$ & $900_{-300}^{+200}$ & 7 \\
\hline NGC 7590 & 0.0053 & $\mathrm{n}$ & $33 \mathrm{~A}$ & 0.168 & 40.02 & 27 & 1.2 & $<20.964$ & $\ldots$ & 49 \\
\hline Was 49b & 0.063 & $\mathrm{y}$ & $54 \mathrm{~L}$ & 33.8 & 42.51 & 57 & 0.63 & 22.799 & $620 \pm 250$ & 21 \\
\hline
\end{tabular}

Note. - The Sy2s with footnote ${ }^{*}$ denote the PBL was detected in later spectropolarimetric observation. Telescope: $\mathrm{C}=\mathrm{CTIO}(4 \mathrm{~m}), \mathrm{P}=$ Palomar (5m), K = Keck (10m), L = Lick (3m), S = Subaru (8.2m), E = ESO (3.6m), A = AAT (3.9m).

References. - (1) Young et al. 1996; (2) Lumsden et al. 2001; (3) Antonucci et al. 1985; (4) Smith \& Wilson 2001; (5) Cappi et al. 2006; (6) Imanishi \& Terashima et al. 2004; (7) Guainazzi et al. 2005a; (8) Guainazzi et al. 2005b; (9) tartarus.gsfc.nasa.gov; (10) Inglis et al. 1993; (11) Bianchi et al. 2003; (12) Levenson et al. 2005; (13) This work; (14) Bianchi et al. 2005a; (15) Tran 2001; (16) de Grijp et al. 1992; (17) Oliva et al. 1994; (18) Ueno et al. 2000; (19) Colina et al. 1991; (20) Miller et al. 1990; (21) Awaki et al. 2000; (22) Dahari \& De Robertis 1988; (23) Moran et al. 2000; (24) Murayama et al. 1998; (25) Matt et al. 2003; (26) Tran 1995; (27) Storchi-Bergmann et al. 1995; (28) Cruz-Gonzalez et al. 1994; (29) Alexander et al. 2000; (30) Iwasawa et al. 2004; (31) Ho et al. 1997; (32) Tran 2003; (33) Heisler et al. 1997; (34) Veilleux et al. 1995; (35) Vaceli et al. 1997; (36) Prieto et al. 2002; (37) de Robertis \& Osterbrock 1986; (38) Storchi-Bergmann \& Pastoriza 1989; (39) Polletta et al. 1996; (40) Maiolino et al. 1998; (41) Whittle et al. 1992; (42) Moran et al. 2001; (43) Mulchaey et al. 1994; (44) Acker et al. 1991; (45) Bianchi et al. 2005b; (46) Kay \& Moran 1998; (47) Fraquelli et al. 2003; (48) Lonsdale et al. 1992; (49) Bassani et al. 1999; (50) Vignali \& Comastri 2002; (51) Matt et al. 2004; (52) Guainazzi et al. 2004; (53) Franceschini et al. 2003; (54) Tran et al. 1992; (55) Terlevich et al. 1991; (56) Masanori et al. 2004; (57) Moran et al. 1992; (58) Lumsden et al. 2004; (59) Dewangan et al. 2003; (60) Dewangan \& Griffiths 2005; (61) Kollatschny et al. 1983; (62) Risaliti et al. 2000; (63) Levenson et al. 2006; (64) Storchi-Bergmann et al. 1992; (65) Matsumoto et al. 2004; (66) Ruiz et al. 1994; (67) Dadina \& Cappi 2004; (68) Duc et al. 1997; (69) Pernechele et al. 2003; (70) Braito et al. 2003. 
Table 2. Best-fit parameters for the X-ray spectral analysis

\begin{tabular}{|c|c|c|c|c|c|c|c|c|c|}
\hline $\begin{array}{l}\text { Name } \\
(1)\end{array}$ & $\begin{array}{l}\text { XMM/Chandra obs. date } \\
(2)\end{array}$ & $\mathrm{N}_{\mathrm{H}}$ & $\Gamma_{(4)}$ & $\begin{array}{r}\text { Center Energy } \\
(5)\end{array}$ & $\begin{array}{r}\mathrm{EW}(\mathrm{Fe} \mathrm{K}) \\
(6)\end{array}$ & $\begin{array}{r}f_{\mathrm{s}}(\%) \\
(7)\end{array}$ & $\begin{array}{c}C / d o f \\
(8)\end{array}$ & $\begin{array}{r}\mathrm{F}_{2-10 k e V} \\
(9)\end{array}$ & $\begin{array}{l}\mathrm{T} \\
(10)\end{array}$ \\
\hline NGC 34 & XMM 2002 Dec 22 & $>100$ & $2.38 \pm 0.1$ & $6.4^{\dagger}$ & $<321$ & $\ldots$ & $362 / 448$ & 0.23 & 0.03 \\
\hline NGC 3982 & XMM 2004 Jun 15 & $>100$ & $3.74_{-1.6}^{+1.8}$ & $6.4^{\dagger}$ & $6310_{-3170}^{+3500}$ & $\ldots$ & $109 / 135$ & 0.057 & 0.086 \\
\hline NGC 5728 & Chandra 2003 Jun 27 & $78_{-14}^{+15}$ & $2.73 \pm 0.17$ & $6.39 \pm 0.03$ & $1100_{-270}^{+320}$ & $0.4 \pm 0.03$ & $299 / 300$ & 1.33 & 0.17 \\
\hline NGC 6552 & XMM 2002 Oct 18 & $71_{-10}^{+44}$ & $2.8_{-0.13}^{+0.37}$ & $6.4^{\dagger}$ & $1408_{-883}^{+668}$ & $0.75_{-0.22}^{+0.09}$ & $121 / 166$ & 0.43 & 0.27 \\
\hline NGC 7172 & XMM 2002 Nov 18 & $8.7 \pm 0.57$ & $1.49 \pm 0.13$ & $6.4^{\dagger}$ & $40 \pm 30$ & $0.54 \pm 0.1$ & $463 / 495$ & 22 & 550 \\
\hline Mrk 573 & XMM 2004 Jan 15 & $>100$ & $3.7_{-0.10}^{+0.08}$ & $6.4^{\dagger}$ & $2800_{-1220}^{+1820}$ & $\ldots$ & $394 / 399$ & 0.12 & 0.07 \\
\hline IRAS 01475-0740 & XMM 2004 Jan 21 & $0.39_{-0.02}^{+0.04}$ & $2.06 \pm 0.06$ & $6.4^{\dagger}$ & $130(<344)$ & $\ldots$ & $631 / 776$ & 0.75 & 0.92 \\
\hline Mrk 1066 & Chandra 2004 Jul 18 & $>100$ & $2.75_{-0.07}^{+0.17}$ & $6.34_{-0.06}^{+0.17}$ & $1120_{-650}^{+850}$ & $\ldots$ & $497 / 505$ & 0.23 & 0.05 \\
\hline
\end{tabular}

Note. - Col. (1): galaxy name. Col. (2): Observation date. Col. (3) power-law photon index. Col. (4): measured absorption column density, in units of $10^{22} \mathrm{~cm}^{-2}$. Col. (5): the Fe line energy in units of keV. Col. (6): the Fe line equivalent width in units of eV. Col. (7): scattering fraction of the soft component. Col. (8): C statistic and number of degrees of freedom (dof). Col (9): the fitted 2-10 Kev flux in units of $10^{-12} \mathrm{erg} \mathrm{s}^{-1} \mathrm{~cm}^{-2}$. Col (10) T: the ratio of $\mathrm{F}_{2-10 \mathrm{keV}} / \mathrm{F}_{[\mathrm{O} \mathrm{III}]} \cdot{ }^{\dagger}$ : fixed. 
Table 3. A summary of the Statistical Properties for Sy2s

\begin{tabular}{lcclr}
\hline \hline Parameters & Sy2s with PBL & Sy2s without PBL & Note & $\mathrm{p}_{\text {null }}^{a}$ \\
\hline $\log (\mathrm{T})$ & $-0.087 \pm 0.145$ & $-0.342 \pm 0.217$ & total & $25 \%$ \\
$\log (\mathrm{T})$ & $-0.201 \pm 0.138$ & $-0.87 \pm 0.186$ & luminous & $0.9 \%$ \\
$\log (\mathrm{T})^{b}$ & $-0.093 \pm 0.144$ & $-0.436 \pm 0.199$ & total & $13.5 \%$ \\
$\log (\mathrm{T})^{b}$ & $-0.203 \pm 0.137$ & $-0.899 \pm 0.179$ & luminous & $0.68 \%$ \\
$\log [\mathrm{EW}(\mathrm{Fe})]$ & $2.654 \pm 0.107$ & $2.85 \pm 0.127$ & total & $18.6 \%$ \\
$\log [\mathrm{EW}(\mathrm{Fe})]$ & $2.626 \pm 0.107$ & $2.999 \pm 0.066$ & luminous & $4.67 \%$ \\
$\log \left(\mathrm{N}_{\mathrm{H}}\right)$ & $23.755 \pm 0.190$ & $23.852 \pm 0.274$ & total & $66.5 \%$ \\
$\log \left(\mathrm{N}_{\mathrm{H}}\right)$ & $23.739 \pm 0.212$ & $24.428 \pm 0.192$ & luminous & $7.7 \%$ \\
$\log \left(\mathrm{L}_{[\mathrm{O}} \mathrm{III}\right]$ & $42.099 \pm 0.13$ & $41.338 \pm 0.188$ & total & $0.34 \%$ \\
$\log \left(\mathrm{L}_{[\mathrm{O}} \mathrm{III}\right]$ & $42.198 \pm 0.121$ & $41.991 \pm 0.157$ & luminous & $30.6 \%$ \\
$\log (\mathrm{z})$ & $0.020 \pm 0.003$ & $0.017 \pm 0.004$ & luminous & $29.4 \%$ \\
\hline
\end{tabular}

Note. - ${ }^{a}$ the possibility pnull is for the null hypothesis that the two distributions are drawn at random from the same parent population. ${ }^{b}$ including 8 hard X-ray upper limits stated in $\S 2$. When there are censored data, we use Gehan's generalized Wilconxon test-permutation variance (GGW test, one kind of ASURV test). "total": all Sy2s with X-ray data in the sample, "luminous": for Sy2s with $\mathrm{L}_{[\mathrm{O} \text { III] }}>10^{41} \mathrm{erg} \mathrm{s}^{-1}$ only. 